\title{
Viral Hepatitis A to E: An Update in 2010
}

\author{
Masatoshi Kudo \\ Department of Gastroenterology and Hepatology, Kinki University School of Medicine, Osaka, Japan
}

Viral hepatitis is still one of the major health-care problems worldwide, since hepatitis B and C can cause the potentially fatal conditions of hepatocellular carcinoma (HCC) and/or liver failure. Similarly, hepatitis A is still a serious problem in Asia and, interestingly, hepatitis $\mathrm{E}$ is re-emerging as a topic of medical discussion, since it causes fulminant hepatitis even in developed countries like Japan.

The 6th Korea-Japan Liver Symposium was held in Kyongju (Korea) on July 18 and 19, 2009, to focus on and discuss current and emerging topics related to viral hepatitis. The symposium began with eye-opening lectures by some of the world's leading researchers, followed by extensive discussion. This issue of Intervirology selects the most important articles presented to this congress.

\section{Hepatitis A}

Due to improved living conditions and subsequent changes in hepatitis A epidemiology, the burden of this disease is increasing in many regions. Recently, Korea has faced a large, community-wide outbreak of hepatitis A, which has prompted a vaccination program [1].

Hepatitis A infection is caused by the hepatitis A virus (HAV), which is transmitted through the fecal-oral route. Lifelong protective antibodies are present after infection. The prevalence of anti-HAV in the 10-50-year age range has declined rapidly during the last three decades. As a result, this age group has a high risk for HAV infection, and clinically overt acute hepatitis A is increasing in adolescents and adults. It is well established that the severity of the disease is related to the age of the patients. The clinical features and the epidemiological shift of HAV underscores the importance in Korea, as well as in other countries with similar issues, of childhood vaccination and consideration of catch-up vaccination for adolescents and adults as well as targeted vaccination for individuals at increased risk for infection or its complications.

An active campaign for universal childhood HAV vaccination should be continued and catch-up vaccination, which is directed at people between 10 and 50 years of age, should be considered. Additionally, conventional high-risk groups and persons more vulnerable to developing fulminant hepatitis - such as travelers to highly endemic areas, patients medicated with clotting factors and patients with chronic liver disease - should be vaccinated. To provide evidence-based recommendations for HAV vaccination, an urgent nationwide survey of HAV seroepidemiology as well as regional surveys and studies of the cost-effectiveness of vaccination of each vaccination strategy are needed.

The clinical spectrum of HAV infection ranges from asymptomatic infection to fulminant hepatitis. Clinical manifestations depend on the age of the host; in other words, less than $30 \%$ of infected young children are symptomatic, while about $80 \%$ of infected adults manifest as severe hepatitis with remarkably elevated serum aminotransferases. Fulminant hepatitis is rare, with a reported incidence from 0.015 to $0.5 \%$. Atypical manifesta-

\section{KARGER}

Fax +4161306 1234 E-Mail karger@karger.ch www.karger.com
(ㄷ) 2010 S. Karger AG, Basel

0300-5526/10/0531-0005\$26.00/0

Accessible online at:

www.karger.com/int
Masatoshi Kudo, MD, PhD

Department of Gastroenterology and Hepatology

Kinki University School of Medicine

377-2 Ohno-Higashi, Osaka-Sayama, Osaka 589-8511 (Japan)

Tel. +81 72366 0221, Fax +81 72367 2880, E-Mail m-kudo@ med.kindai.ac.jp 
tions include relapsing hepatitis and prolonged cholestasis, and complicated cases with acute kidney injury have been reported [2].

Management of hepatitis A includes general supportive care, and critical decisions regarding liver transplantation await further studies on prognostic predictors. Fundamental management of hepatitis A is active vaccination. However, a vaccination program should be adapted to the regional situation, according to differing epidemiology and disease burden [2].

\section{Hepatitis B}

Hepatitis B virus (HBV) vaccination has effectively reduced the acute and chronic infection rates in recent years. Since 1983, HBV vaccination has been recommended for all neonates in many countries, including Korea. Before the introduction of the HBV vaccination program, approximately $8 \%$ of the general Korean population tested positive for HBsAg. The percentage of vaccinated infants has surpassed $98.9 \%$ since 1990 . The $\mathrm{HBsAg}$ carrier rate in the general population decreased to $3.7 \%$ in 2007 . In particular, the prevalence of $\mathrm{HBsAg}$ decreased to $0.44 \%$ in teenagers and $0.2 \%$ in children younger than 10 years. In addition, administration of the HBV vaccine may have reduced the risk of HCC among adults. Despite the administration of hepatitis B immunoglobulin and the HBV vaccine to children with HBsAg-positive mothers, the failure rate of HBV immunoprophylaxis was $4.2 \%$ in 2008 . In Korea, there have been no reported cases of HBV surface gene variants such as G145R.

HBV vaccination has effectively reduced the infection and chronicity rates of HBV and related complications. The overall prevalence of $\mathrm{HBV}$ vaccination has exceeded $99 \%$ since 1990 . However, acute HBV infection may still occur in unvaccinated and uninfected adults. In those cases, catch-up vaccination will be needed. Despite considerable effort to reduce HBV infection via the universal vaccination of all newborn and school-age children, a large proportion of the population was previously infected with HBV and still harbors the virus. The prevalence of HBV carriage in Korea declined after the introduction of a universal HBV vaccination program. Korea is now classified as an area of intermediate endemicity for HBV. The prevention of complications such as cirrhosis and HCC in infected individuals requires appropriate therapeutic agents [3].
The goals of antiviral therapy in patients with chronic hepatitis B are long-lasting suppression of HBV DNA, normalization of serum alanine aminotransferase (ALT) and prevention of progression of chronic liver disease to liver cirrhosis, HCC and liver-related death. Even though substantial advances have been made in the treatment of chronic hepatitis $\mathrm{B}$ in the past decade with the use of oral nucleoside/nucleotide analogues (NAs), emergence of antiviral resistance is the most important factor in treatment failure for chronic hepatitis B. Therefore, to prevent antiviral resistance, development of antiviral agents that act with different mechanisms and at different sites (the paradigm of combination therapy for HIV management) is needed. However, such antiviral agents are unlikely to become available in the near future. Therefore, an understanding of the molecular basis of NA resistance and an optimal use of NAs are important for the time being. To minimize the emergence of drug resistance using currently available antiviral agents, physicians should avoid unnecessary therapy. Once initiated, an antiviral agent should suppress viral replication as quickly and completely as possible. Drug resistance should be continuously monitored. However, once antiviral resistance develops, prompt combination therapy should be initiated [4].

\section{Hepatitis C}

HCC is one of the leading cause of death from cancer worldwide. Hepatitis $\mathrm{C}$ virus (HCV) is a major cause of HCC, accounting for $70 \%$ of all HCC cases in Japan. HCV genotype $1 \mathrm{~b}$, the most prevalent subtype in Japan, started to spread in the 1930s among injecting drug users or through medical procedures such as blood transfusion and use of contaminated syringes. The prevalence of HCV infection is much lower in the current younger generation compared with that in the older generation, particularly those aged $>55$ years old $(0.1-0.2$ vs. $\geq 2 \%$ ). Therefore, the total number of patients with HCV infection is expected to decrease, even though sporadic HCV transmission is mainly seen among young injecting drug users. Of note, $\mathrm{HCV}$ genotype 2 seems to be spreading among young drug users, but the response to antiviral therapy in these patients is better than that in older patients, irrespective of the genotype. Although the number of patients who die because of HCC has steadily increased over the last 50 years, the incidence of HCC is now decreasing, mainly because of the decreased prevalence of HCV-related HCC [5]. 
The spread of HCV infection in Japan started in the 1930 s, and widespread dissemination of the virus has occurred since then. The risk of iatrogenic HCV transmission has been almost eliminated; however, sporadic HCV transmission still occurs. The prevalence of HCV infection in the younger generation is extremely low and the total number of HCV patients is expected to decrease. Although the number of patients who die of HCC has steadily increased because of the dissemination of $\mathrm{HCV}$ infection in the past, it is estimated to decrease in the near future because of the decrease in rates of HCV-related HCC [5].

New drugs that can be used in combination with interferon (IFN) are being actively developed. Also, attempts are being made to physically remove HCV particles from the blood. Granulocyte apheresis, plasma exchange and hemofiltration have been applied to HCVinfected patients for the treatment of cryoglobulinemia and vasculitis, modalities shown to reduce HCV RNA in the blood during treatment. The mechanisms of the clinical results of plasmapheresis have been described, whereby $\mathrm{HCV}$ in the blood is related to the effects of IFN therapy that could be enhanced by removing the virus from the blood. Low-density lipoprotein cholesterol apheresis and plasma exchange in hypercholesteremic patients with $\mathrm{HCV}$ infection reduces the quantity of HCV RNA in the blood of some patients. Hemodialysis, hemofiltration and peritoneal dialysis in chronic dialysis patients infected with HCV significantly lower HCV RNA levels in the blood. Combined granulocyte apheresis with IFN therapy for chronic hepatitis $\mathrm{C}$ and the prerequisite for early reduction of the virus in the treatment of chronic hepatitis $\mathrm{C}$ are essential. Thus, the potential effectiveness of IFN therapy combined with early physical removal of the virus is of particular interest [5].

Double-filtration plasmapheresis (DFPP) was approved in April 2008 in Japan for the retreatment of chronic hepatitis $\mathrm{C}$ patients with genotype $1 \mathrm{~b}$ and high viral loads, whose HCV was not eradicated by earlier IFN therapy or by pegylated IFN plus ribavirin (PEG-IFN/ $\mathrm{RBV}$ ) combination therapy. In the current issue, Kim et al. [6] assessed the early viral dynamics of 9 patients with non-sustained virological response (non-SVR) to combination therapy. The overall viral dynamics of DFPP plus IFN treatment with or without RBV for 4 weeks showed a reduction of $\geq 1 \log$ in $22 \%$ ( 2 of 9 patients), $55.6 \%$ (5/9), $77.8 \%(7 / 9)$ and $77.8 \%(7 / 9)$ in the viral load at $24 \mathrm{~h}, 1$ week, 2 weeks and 4 weeks after the start of treatment. In contrast, DFPP plus consecutive intravenous IFN- $\beta$ for 4 weeks reduced the viral load by $\geq 1 \log$ in $33 \%(2 / 6), 50 \%$
(3/6), 83.3\% (5/6) and 83.3\% (5/6) at $24 \mathrm{~h}, 1$ week, 2 weeks and 4 weeks. The viral load declined by $\geq 2 \log$ in $50 \%$ $(3 / 6)$ at 4 weeks after the start of treatment. DFPP plus consecutive intravenous IFN- $\beta$ for 4 weeks is a promising treatment for non-SVR patients [6].

The prerequisite for early virological response (EVR: indicating negative HCV RNA at 12 weeks) has been emphasized in predicting SVR and non-SVR in chronic hepatitis $C$ patients undergoing IFN treatment; those who do not reach EVR fail to respond to further therapy. Treatment discontinued in patients not reaching EVR would reduce drug costs by more than $20 \%$; consequently, early confirmation of viral reduction after initiating antiviral therapy for chronic hepatitis $\mathrm{C}$ is highly desirable [6].

From the above considerations, DFPP plus consecutive intravenous IFN- $\beta$ treatment for 4 weeks is a promising regimen for non-SVR patients with genotype $1 \mathrm{~b}$ and high viral loads who have been previously treated with PEG-IFN/RBV therapy [6].

Sasase et al. [7] investigated whether SVR and nonSVR by chronic hepatitis C patients to PEG-IFN/RBV combination therapy are distinguishable by viral factors such as the IFN/RBV resistance-determining region (IRRDR) and by on-treatment factors through new indices such as the rebound index (RI). The first RI (RI-1st: the viral load at week 1 divided by the viral load at $24 \mathrm{~h}$ ) and the second RI (RI-2nd: the viral load at week 2 divided by the viral load at $24 \mathrm{~h}$ ) were calculated, and the subject patients were divided into 3 groups based on RI1st and RI-2nd: an RI-A group (RI-1st $\leq 1.0$ ), an RI-B group (RI-1st $>1.0$ and RI-2nd $<0.7$ ) and an RI-C group (RI-1st $>1.0$ and RI-2nd $\geq 0.7$ ). The SVR rate was $71.4 \%$ $(10 / 14)$ in the RI-A group, $46.2 \%$ (6/13) in the RI-B group and $20.0 \%(3 / 15)$ in the RI-C group ( $\mathrm{p}=0.005$ between the RI-A group and the RI-C group). In IRRDR $\geq 6$ and IRRDR $\leq 5$, the SVR rate was $81.3 \%(13 / 16)$ and $23.1 \%$ $(6 / 26)(p=0.0002)$, respectively. By combining RI and IRRDR as a predicting factor, the SVR rate was $87.5 \%$ $(7 / 8)$ in the RI-A group (IRRDR $\geq 6)$ and $7.7 \%(1 / 13)$ in the RI-C group (IRRDR $\leq 5)(\mathrm{p}=0.0003)$. Therefore, IRRDR combined with RIs is the quite promising predictor for SVR and non-SVR. With the aid of RIs and IRRDR, a more effective PEG-IFN/RBV treatment could be within reach [7].

Another approach to increase the SVR rate, the extension of treatment duration, has been proposed for late virological responders infected with HCV genotype 1 and high viral load. However, the effectiveness of extended treatment in patients whose serum HCV RNA becomes undetectable later than 24 weeks of treatment (ultra-late 
virological responder; ULVR) has not yet been determined. Ueda et al. [8] reported that among a total of 130 patients infected with HCV genotype 1 and high viral load treated with PEG-IFN/RBV combination therapy, 10 ULVR received extended combination treatment beyond 48 weeks. The duration of the combination treatment for ULVR ranged between 59 and 119 weeks and the mean duration was 80 weeks. Although the majority of ULVR were older female patients ( $\geq 60$ years; older age and female sex both being factors related to poor therapeutic response), 8 patients (80\%) achieved SVR. The SVR rate correlated well with the duration of the treatment. Five ULVR achieved SVR when treatment was continued until serum HCV RNA remained undetectable for longer than 48 weeks. From these observations it is concluded that the extended duration of PEG-IFN/RBV combination treatment is a possible strategy to improve treatment response in HCV genotype 1 infection, even for ULVR. The extension of the treatment does not seem to increase side effects or the rate of dose reductions, and treatment should be continued until the serum HCV RNA remains undetectable for at least 24 weeks and, if possible, for longer than 48 weeks during the course of treatment [8].

Recently, the significance of serum ferritin levels in PEG-IFN/RBV combination therapy for chronic hepatitis $\mathrm{C}$ has become of interest. Yada et al. [9] examined the correlation of serum ferritin level with serum ALT levels during therapy and response to the therapy. A total of 175 patients with chronic hepatitis $\mathrm{C}$ received the combination therapy. Correlation between serum ferritin levels and serum ALT levels at 12 and 24 weeks of therapy were examined. Differences in serum ferritin levels during therapy between patients with SVR and non-SVR were also examined. The authors found that only 24 (13.7\%) and $20(11.4 \%)$ patients showed elevated serum ALT levels $(\geq 70 \mathrm{IU} / \mathrm{l})$ at 12 and 24 weeks of therapy, respectively. There was no correlation between serum ferritin levels and ALT levels. Ninety-five (54.3\%) of 175 patients achieved SVR. Serum ferritin levels increased dramatically in both SVR and non-SVR groups after starting the therapy and were significantly higher in the SVR group throughout the therapy. Therefore, they concluded that serum ferritin level increases during PEG-IFN/RBV combination therapy; however, it did not correlate with either serum ALT level or the total dose of RBV. Higher serum ferritin levels during combination therapy are associated with a favorable therapeutic response, which has not been reported before.

Enomoto et al. [10] searched HCV genetic elements determining the early response to PEG-IFN/RBV thera- py using HCV genome-wide analysis. From a total of 88 chronic hepatitis $\mathrm{C}$ patients with $\mathrm{HCV}-1 \mathrm{~b}$ treated with PEG-IFN/RBV, the whole HCV amino acid sequence was determined and analyzed according to the viral response during the treatment. Mutations in NS5A-ISDR are associated with rapid viral response at week 4 , and the core arginine70glutamine (R70Q) mutation is associated with no early viral response at week 12 , revealing that core 70 and NS5A are the most important factors determining the virological kinetics during PEG-IFN/RBV therapy. Viral genome-wide analysis is a promising tool for elucidating the unknown viral factors involved in different pathological pictures, such as disease progression [10].

\section{Hepatitis E}

The epidemiology of acute viral hepatitis (AVH) is dynamic and is affected by many factors including hygiene, socioeconomic status and vaccination coverage. A total of 4,302 cases of AVH were sequentially studied on a nationwide scale between 1980 and 2008. Acute hepatitis A (AHA), acute hepatitis B (AHB), acute hepatitis C (AHC), and non- $\mathrm{A}$, non-B and non- $\mathrm{C}$ (non- $\mathrm{ABC}$ ) hepatitis accounted for 1,583 (36.8\%), 1,197 (27.8\%), 359 (8.3\%) and $1,163(27.0 \%)$ of all AVH. The proportions of AHA, AHB, AHC and non-ABC were approximately 40, 25, 10 and $25 \%$, respectively, between 1980 and 1995 . The proportions were approximately $30,30,10$ and $30 \%$ between 1996 and 2003, and shifted to approximately 10, 40, 10, and $40 \%$ in the last 5 years. The number of AHB caused by genotype $\mathrm{A}$, which is not indigenous to Japan, was 6.0\% between 1991 and 1996 and markedly increased after 2000 to reach $52 \%$ in 2008 . Autochthonous acute hepatitis $\mathrm{E}$ (AHE) accounted for $10-15 \%$ of non-ABC hepatitis after 2002. The etiology of AVH in Japan has been drastically changing. A marked increase of AHB genotype A and constant occurrence of autochthonous AHE require attention, and necessary measures should be taken [11].

HEV is a major cause of acute hepatitis in many developing countries where AHE is an important public health concern. However, cases of sporadic AHE in people with no history of recent travel have been reported in developed regions such as North America, Europe, Japan and Australia. The reporting of such infections together with the availability of more comprehensive molecular and serological data has led to the re-evaluation of HEV epidemiology and the acceptance that autochthonous AHE is a clinical problem in developed countries. Information 
on AHE in non-ABC hepatitis populations in Japan is limited, although there are many reports of sporadic or epidemic occurrence of AHE. The current study also showed the trend of AHE in Japan. AHE constituted 4.9\% (44/896) of non-ABC hepatitis. Although the number of AHE cases (1-6 cases per year) and its ratio to non-ABC hepatitis (0-14.5\%) are not very high and are insignificant, the occurrence of AHE became constant after 2002. Surprisingly, AHE constituted as much as $11.0 \%(25 / 228)$ of non-ABC hepatitis after 2002. The clinical course was generally modest and none of the patients showed severe type of hepatitis, probably because most domestic cases were caused by HEV genotype 3, which has been associated with milder clinical outcome compared to HEV genotype 4 . This phenomenon may reflect the fact that their sentinels involve only a few institutes in Hokkaido, where HEV genotype 4 is endemic. Nevertheless, the trend of AHE requires particular attention, because modes of transmission are still often unknown, even after taking very careful history of eating particular foods such as raw meat of deer, pigs and boars [11].

\section{Fibrosis and Hepatocarcinogenesis by Viral Hepatitis}

FibroScan has many limitations. It cannot be used in patients with ascites, thick subcutaneous fat, narrow intercostals spaces and hepatic atrophy. Real-time elastog- raphy (RTE) does not have such limitations and can be used in almost all patients including those with conditions mentioned above, who have difficulty in using FibroScan. However, RTE requires training to analyze the data and also to scan the patients to obtain reproducible images. To address these issues in RTE, Tatsumi et al. [12] investigated easy-to-use acquisition techniques to reduce inter-observer variability and also to simplify RTE image acquisition.

FibroScan and RTE both correlate highly with the F staging of the liver using biopsy. In particular, RTE is very useful for the differential diagnosis and staging of the liver fibrosis.

More cases need to be evaluated by FibroScan and RTE for liver fibrosis staging and to establish guidelines to minimize unnecessary liver biopsies, which will significantly benefit the patients with chronic liver diseases. Furthermore, RTE might be a very good indicator to predict the incidence rate of HCC in the near future [12].

\section{Disclosure Statement}

The author declares that he has no financial conflict of interest.

\section{References}

1 Kim YJ, Lee HS: Increasing incidence of hepatitis A in Korean adults. Intervirology 2010; 53:10-14.

2 Jeong SH, Lee HS: Hepatitis A: clinical manifestations and management. Intervirology 2010;53:15-19.

3 Park NH, Chung YH, Lee HS: Impacts of vaccination on hepatitis B viral infections in Korea over a 25-year period. Intervirology 2010; 53:20-28.

4 Song BC: How to overcome antiviral-resistant hepatitis B virus? Intervirology 2010; 53:29-38.

5 Chung H, Ueda K, Kudo M: Changing trends in hepatitis $\mathrm{C}$ infection over the past 50 years in Japan. Intervirology 2010;53:39-43.
6 Kim SR, Imoto S, Kudo M, et al: Double-filtration plasmapheresis plus IFN for HCV-1b patients with non-sustained virological response to previous combination therapy: early viral dynamics. Intervirology 2010; 53:44-48.

7 Sasase N, Kim SR, Kudo M, et al: Outcome and early viral dynamics with viral mutation in PEG-IFN/RBV therapy for chronic hepatitis in patients with high viral loads of serum HCV RNA genotype 1b. Intervirology 2010;53:49-54.

8 Ueda T, Chung H, Kudo M, et al: Prolonged PEG-IFN and RBV is effective in patients with HCV genotype 1 and high viral load who achieved virological response later than 24 weeks. Intervirology 2010;53:55-59.
9 Yada N, Kudo M, Chung H, et al: PEG-IFN $\alpha$ / RBV combination therapy for chronic hepatitis $\mathrm{C}$ patients increases serum ferritin level while it improves sustained viral response rate. Intervirology 2010;53:60-65.

10 Enomoto N, Maekawa S: HCV genetic elements determining the early response to peginterferon and ribavirin therapy. Intervirology 2010;53:66-69.

11 Yano K, Tamada Y, Yatsuhashi H, et al: Dynamic epidemiology of acute viral hepatitis in Japan. Intervirology 2010;53:70-75.

12 Tatsumi C, Kudo M, Ueshima K, et al: Noninvasive evaluation of hepatic fibrosis for type $\mathrm{C}$ chronic hepatitis. Intervirology 2010; 53:76-81. 